\title{
(6) OPEN ACCESS \\ Advancing point of care diagnostics for the control and prevention of STIs: the way forward
}

\author{
Igor Toskin, ${ }^{1}$ Karel Blondeel, ${ }^{1,2}$ Rosanna W Peeling, ${ }^{3}$ Carolyn Deal, ${ }^{4}$ James Kiarie ${ }^{1}$
}

'Department of Reproductive Health and Research, World Health Organization, Geneva, Switzerland

2Faculty of Medicine and Health Sciences, Ghent University, Ghent, Belgium

${ }^{3}$ Department of Clinical Research, London School of Hygiene and Tropical Medicine, London, UK

${ }^{4}$ Division of Microbiology and Infectious Diseases, National Institute of Allergy and Infectious Diseases, Rockville, USA

\section{Correspondence to} Dr Igor Toskin, Department of Reproductive Health and Research, World Health Organization, Avenue Appia Geneva, CH 1211, Switzerland; toskini@who.int

Received 30 March 2017 Revised 6 June 2017 Accepted 23 June 2017

\section{(1) crossank}

To cite: Toskin I, Blondeel K, Peeling RW, et al. Sex Transm Infect 2017:93:S81-S88.

\section{ABSTRACT}

WHO recognises the global impact of sexually transmitted infections (STIS) on global public health and individual sexual and reproductive health and well-being. As a component of the WHO Global Health Sector Strategy for the control and prevention of STIS, there has been a growing recognition of the importance of integrating point-of-care tests (POCTs) into overall strategic planning. The process of integrating STI POCTS, in addition to providing a definitive diagnosis and appropriate treatment in a single visit, also includes innovative delivery options, such as on-site delivery, community-based testing (including screening), as well as self-testing at home after purchase of a test online or over-the-counter. WHO organised two technical consultations in May 2014 and July 2015. This article summarises the discussions of the meeting participants on advancing the use of POCTs to control and prevent STIs. The following priorities were identified: the need for pathogens' target discovery; encouragement of multiplexing, miniaturisation, simplification and connectivity; promotion of standardisation of evaluation of new diagnostic platforms across all stages of the evaluation pipeline; the need for an investment case, modelling and scenarios to ensure buy-in among key stakeholders, including developers and the private sector; the need for norms and standards, including guidelines, to support introduction of STI POCTs in programmes; anticipating potential tensions between different parties at the implementation level; and leveraging on the global initiative, Sustainable Development Goals (SDGs)/global health sector STI strategy, to sustain investment in STI POCT programmes. There is a rich pipeline of diagnostic products, but some have stalled in development. An approach to accelerate the evaluation of new diagnostics is to set up a competent network of evaluation sites ahead of time, harmonise regulatory approval processes with development of models to estimate costeffectiveness, informed by better STI data. This should result in accelerating policy development. Although it may be some time before good POCTs can be widely implemented in low resource settings, it is important to be a catalyst for continued development and use of these essential tools as an integral part of both the WHO Global Health Sector Strategy and the agenda for 2030.

\section{INTRODUCTION}

WHO recognises the global impact of STIs on global public health and individual sexual and reproductive health and well-being. As a component of the WHO Global Strategy for the Control and Prevention of STIs, there has been a growing recognition of the importance of integrating point-of-care tests (POCTs) into overall strategic planning. ${ }^{1}$ Advancement of these technologies would allow the delivery of aetiology-based diagnosis to move from centralised laboratory-based testing to a clinic-based approach closer to the setting in which patients seek healthcare. This approach could serve many of the goals in the global strategy and provide patients and clinicians with a rapid diagnosis and a better informed basis for treatment.

The STI POCT concept, in addition to enabling a definitive diagnosis and appropriate treatment in a single visit, also includes innovative delivery options, such as on-site delivery, community-based testing (including screening), as well as self-testing at home after purchase of a test online or over-the-counter. ${ }^{2}$

In support of this concept, WHO organised two technical consultations, one in May 2014 and the other in July 2015. These consultations brought together international experts from all WHO regions to review the progress of POCTs and to identify challenges and gaps that need to be addressed to advance their development and use for the control and prevention of STIs.

During the first consultation, the international expert group (IEG) on STI POCTs was established. These experts reviewed the results of the landscape analysis of promising STI diagnostic technologies and tests, and of systematic reviews on the performance and operational characteristics of STI POCTs. The IEG also defined target product profiles (TPPs) for the development of new STI POCTs and advised on further research.

The objectives of the second consultation were to finalise core protocols for independent evaluation of promising STI POCTs, to consider the implementation of the core protocols in selected countries and to develop a roadmap for the development of new STI POCTs, including the research needs for new product development.

Meeting participants focused on the development of tests for which aetiological diagnosis potentially has the greatest impact: dual HIV/syphilis tests, tests for the detection of Neisseria gonorrhoeae (gonorrhoea), Chlamydia trachomatis (chlamydia) and Trichomonas vaginalis (trichomoniasis), at both urogenital and extragenital sites, as well as tests for human papillomavirus infection. The current article summarises the discussions among the meeting participants to advance the use of POCTs to control and prevent STIs.

\section{GAPS, NEEDS AND PRIORITIES}

Inspired by the global roadmap for STI vaccine development, ${ }^{3}$ discussions were structured along four main development phases necessary for 


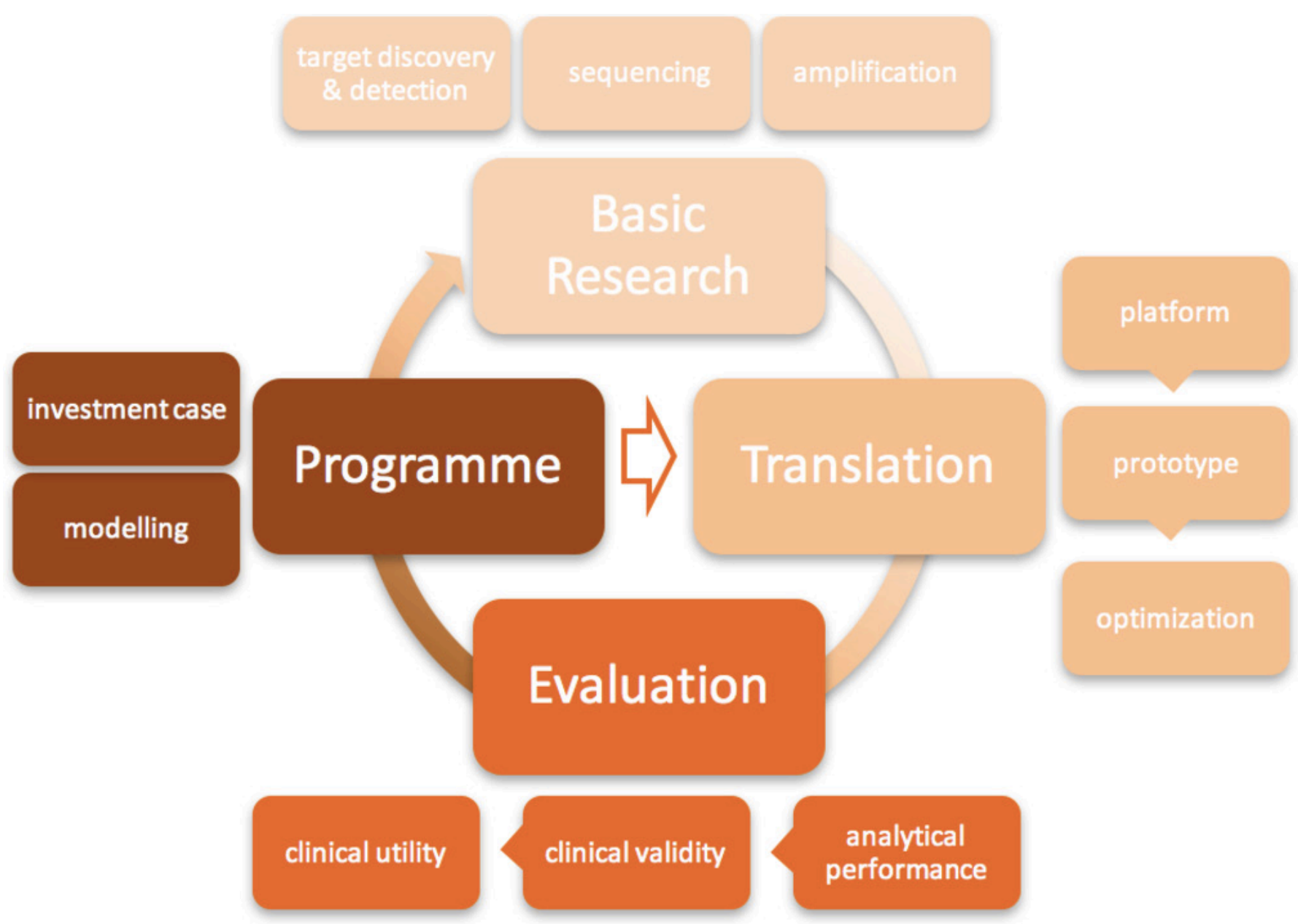

Figure 1 Development cycle of STI POCTs.

advancing products from the laboratory to routine use (figure 1): basic research, translation, evaluation and programmatic implementation. For each phase, meeting participants identified critical gaps in knowledge, current and future needs and formulated important priorities for accelerating development and global uptake of POCTs in public health strategies. To sustain development of all diagnostic tools related to STIs, all participants reiterated the urgent need for better epidemiological data (estimates and surveillance) and for improving the understanding of STI natural history and burden of sequelae, similar to views expressed for the STI vaccine development. ${ }^{4}$ These advances, which will not be discussed in detail here, are needed to break the cycle of a lack of appropriate and affordable STI diagnostic tools resulting in a lack of STI surveillance data. This gap in knowledge, in turn, may limit the interest in STI diagnostics development. Higher quality data on both STI trends and burden are one of the main drivers to advance STI diagnostic development.

Before the participants discussed the way forward for POCTs, it was acknowledged that a range of definitions currently exists for the term, POCTs. In the absence of a universally accepted definition the WHO STI Diagnostics Initiative developed the ASSURED criteria as a benchmark to decide if tests address disease control needs: Affordable, Sensitive, Specific, Userfriendly, Rapid and Robust, Equipment-free and Deliverable to end-users. ${ }^{5}$ These criteria have guided many POCT evaluations and development of new POCTs but have also been criticised for being too subjective and lacking quality assurance on the one hand and on the other for creating artificial restrictions on the concept of POCT, possibly discouraging developers from pursuing their endeavours. ${ }^{6}$ Equipment-free has over the years moved to minimal equipment, meaning portable, simple, mobile devices requiring minimal infrastructural support and affordability has been complemented with cost-effectiveness.

The POCT concept is not only determined by technology but should also be determined by context and the end-users, both providers and patients. Most experts share the vision that STI POCTs should encompass, as a minimum: testing performed on-site during the patient consultation to permit a rapid test result to be generated and used to make an immediate, informed clinical decision. ${ }^{7}$ However, some meeting participants questioned what on-site means, when with the current technologies, home-based testing could be complemented with online counselling and prescription. However, these technologies are currently not available in low-income and middle-income countries, which bear $80 \%-90 \%$ of the global STI burden. ${ }^{8}$

Notwithstanding the lack of a universal definition, the IEG decided on TPPs for five STIs, described earlier in this supplement, which are available on the website of WHO to guide researchers, manufacturers as well as policy makers. ${ }^{9}$

\section{BASIC RESEARCH}

\section{Gaps}

Meeting participants agreed that while current products hold great potential, to maintain a robust development pipeline, basic research is essential to generate new potential avenues and approaches. 
There are gaps in antigen discovery and diagnostic target identification for STIs. For syphilis, markers (specific host or pathogen proteins and antigens or other biomarkers) are lacking to easily and rapidly distinguish acute infection from prior infections, both in adults and infants. For gonorrhoea, there is a need to address antimicrobial resistance (AMR) by developing new rapid methods to determine susceptibility to relevant antibiotics without resorting to culture. ${ }^{10}$ This has the potential to provide patient-specific information in a time frame that allows clinicians to prescribe individualised treatment regimens. ${ }^{11}$ For chlamydia, the meeting participants observed poor sensitivity of immunochromatographic tests to detect chlamydial antigens, especially if these rapid tests are to be used for screening asymptomatic patients with low bacterial loads.

\section{The way forward}

Encourage target discovery

Participants suggested the development of a TPP for POCTs specifically for the elimination of mother-to-child transmission of syphilis. There is a need for a novel marker of active syphilis that can be detected using easy, low-tech, low-cost and effective technologies to replace the detection of non-treponemal antibody (reagin) as a proxy of active syphilis, given the dilemma whether to repeat treatment for patients with persistent non-treponemal antibody. Identifying new markers for active syphilis is a high-risk undertaking, it should however not be neglected by developers, as it is a critical tool for the elimination of mother-to-child transmission of syphilis and management of the 'serofast' patient. Both the utility of absolute titres and comparison of titres over time, and between the baby versus the mother, should be considered.

Participants recommended the identification of genetic markers to predict gonococcal resistance/susceptibility, including nucleic acid targets, against recommended therapeutic agents for gonorrhoea. The frequency of potential diagnostic targets in different gonococcal genotypes and populations should be investigated. Current technologies are complicated, requiring multiple steps, trained personnel and may take more than 48 hours to perform. The novel resistance markers should be incorporated into a POCT to track resistance.

\section{Encourage integration and miniaturisation of sample preparation and target detection}

As was pointed out in the landscape of POCTs in this supplement, sample preparation and target detection are two key processes in diagnostics. 'Next-generation' technologies, such as microfluidics and nanotechnology, enabling the integration and miniaturisation of both processes, should be fully supported to overcome these barriers. ${ }^{12}$ Although miniaturisation allows complex technologies to be used at POC, it should be noted that the smaller sample size required for these nanoscale reactions poses a risk that test sensitivity would be poor unless the diagnostic target is present in ample amounts. ${ }^{13}$

The AMR opinion paper in this supplement shows that microfluidic and nanotechnology platforms provide a basis to develop molecular POC-AMR diagnostics. However, particularly for gonorrhoea, more knowledge is required to assess which antibiotic classes lend themselves to a genotypic POC-AMR approach. It is difficult to estimate the potential clinical and epidemiological impact of using a genotypic approach when genotypic-phenotypic relationships for N. gonorrhoeae are not well understood. ${ }^{14}$

It could be beneficial to improve and promote non-invasive sample collection where possible, for example, urine among men and women in the case of urethrogenital infections, and saliva in case of systemic infections like syphilis, without jeopardising the analytical performance of STI POCTs. Diagnostic platforms should be calibrated according to the quantity of pathogen targets in samples in order to ensure acceptable levels of sensitivity and specificity.

\section{Promote international and multisectorial research cooperation}

To enable research in low-resource settings, participants suggested the creation of biobanks to gather available well-characterised specimens that can be accessed by various stakeholders.

The knowledge of conserved diagnostic target sequences across the globe could be improved by expanding genomic sequence databases.

Although the field of STI POCTs is advancing, initiatives to prompt the uptake of novel technologies have been scarce compared to the POCT field in other areas in clinical medicine. ${ }^{15} \mathrm{~A}$ literature review should be conducted to identify promising crossover initiatives, both for STIs and emerging infectious diseases.

\section{TRANSLATION \\ Gaps}

The two main gaps in the translation of basic research to prototype products are interrelated. While there is a lack of multiplex platforms that combine the detection of several STIs into a single POCT, and potentially other infectious diseases, many of the current and future technologies will not be accessible or affordable for patients in the developing world.

The participants felt that not all potentially viable basic research is translated to products by diagnostic companies, and when it is translated, there is often a disconnect between the aims of the companies and the preferences of the end-users, both patients and providers. These are very much linked and should be directly informed by the stage of implementation and programming.

The IEG also pointed to the underuse of the enormous potential that connectivity technology currently offers. ${ }^{16}$

\section{The way forward}

Prioritise the development of miniaturised multiplexable STI POCT platforms

The development of a POCT that simultaneously screens for multiple pathogens from a single specimen should be prioritised because the same clinical symptoms can be caused by infections by many aetiological agents and mixed infections are common. ${ }^{12}$ Moreover, disease and therapeutic response often involve interplay between many biological processes. ${ }^{13}$ Multiplexing could also potentially increase throughput and data generation while simplifying formats and decreasing the time and cost required to operate tests, which makes them more fit for use in low- and middle-income settings than current POCT platforms.

The IEG stressed the need for further developing next-generation platforms and prototypes to miniaturise the products, their footprints and turnaround time and combining them to achieve multiplexing optimisation. Target combinations should be optimised to improve global strain coverage while reducing any signal interference. The possibility of having a multiplex pregnancy/HIV/syphilis POCT was raised.

One of the very promising prototypes uses microfluidics and lab-on-chip technology, capable of detecting major causes of STIs simultaneously at very low cost per test. ${ }^{17}$ It is, however, not yet commercially available nor widely evaluated. Another promising approach, which combines both spatial and spectral multiplexing, uses quantum dots, potentially increasing the multiplexing 
capabilities of immunoassays. ${ }^{13}$ Recent developments in paperbased microfluidic assays together with novel assay formats have also unprecedentedly expanded the number and complexity of tests that can be conducted using low-cost and disposable POCT kits, particularly in the developing world. ${ }^{18}$ A more comprehensive overview of the next-generation technologies can be found in the landscape article in this supplement.

\section{Include researchers, manufacturers and end-users in the} translational phase of POCTs

The meeting participants acknowledged that in most settings, few appropriate tests are available. Part of the reason being that the scientists and engineers who design these tests, and the companies who develop and market them, are usually based in affluent countries and are often unaware of settings and the real needs of the end-users in the developing world. ${ }^{19}$ Bringing them together with end-users, providers and patients, in the translational stage of POCT development creates an opportunity for proactive problem-solving, discussions about trade-offs and device design. ${ }^{20} 21$ In addition, used to working with few resources and local healthcare professionals might come up with simple yet innovative solutions to challenges around cost-effectiveness.

The IEG stressed the need for more qualitative studies in different settings and populations, to assess end-users' requirements. ${ }^{22}$

\section{Capitalise on the advances in connectivity for STI-surveillance,} testing uptake and quality control of STI POCTs

If an STI POCT comes with a reader and is introduced in a whole region or country, connectivity may be used for STI surveillance purpose. $^{23}$

Quality control is not often included in the translation stage of POCT development. Reader devices should be an asset for enhancing quality control programmes. If data are not coming in, programme managers can immediately investigate the reason (personnel, supplies, treatment stock-outs, etc) and initiate corrective action. Connectivity solutions can be leveraged to turn system stresses into increasing efficiency.

The meeting participants agreed on the need of AMRresearch in $N$. gonorrhoeae to capitalise on the fact that mobile phones are widespread globally, even in low- and middle-income settings, although not all. Mobile phones and smartphone applications could facilitate reading POCT test results, promote uptake and increase follow-up. STI apps might lead to more testing. ${ }^{24}$ The fact that current STI apps have a marked variation in content, quality and accuracy, some even contain potentially harmful information, ${ }^{25}$ calls for the need to invest in a free, good quality e-health approach to sexual health and well-being, which could complement and reinforce the introduction of STI POCTs.

The IEG also emphasised the need of making TTPs for self-administered, home-based STI diagnostic tools, which can be purchased online or through apps. ${ }^{26}$

\section{EVALUATION \\ Gaps}

The IEG recognised the lack of independent evaluations of currently available POCTs, both laboratory-based and clinic-based, and utility evaluations. In particular, the test performance on pharyngeal and rectal swabs for gonorrhoea, chlamydia and trichomoniasis is lacking, since most companies do not have an indication for their tests to be used on these specimens. Little is also known about the cost-effectiveness of pooling specimens for low-prevalence settings. There is also a lack of clinic-based and utility evaluations in key populations, for which STI POCTs seem to be particularly appropriate, and in low- and middle-income settings. In addition, as the reviews in this supplement indicate, the quality of evaluations undertaken in the past has been very uneven. For example, reference testing is often problematic. ${ }^{27}$

\section{The way forward \\ Promote standardised high-quality laboratory, clinic-based and utility evaluations of STI POCTs}

The IEG welcomed the initiative of WHO to prepare core protocols for laboratory, clinic-based and utility evaluation of STI POCTs and indicated those STIs for which POCTs are most urgent and the populations in greatest need of them. The research priorities for diagnostic tests in a public health setting are based on the main challenging areas in terms of STI control from a diagnostic perspective. Linking the discussion on STI POCTs to STI case management as well as STI control and prevention in key populations, the following priorities were identified:

- Chlamydia/trichomoniasis: high prevalence, understanding the purpose of regular screening in women at risk;

- Gonorrhoea: increase of resistance in N. gonorrhoeae, increasing the need for scaling up regular screening of women at high risk;

- Chlamydia/gonorrhoea/trichomoniasis: case management vaginal discharge syndrome needs to optimise case management through introduction of testing;

- Chlamydia/gonorrhoea: screening in men who have sex with men (MSM) in extragenital anatomic sites: using pharyngeal and anorectic swabs;

- Dual HIV/syphilis: screening in antenatal care (ANC) as well as sex workers and MSM.

These protocols have in the mean time been approved by the Research Project Review Panel of the Reproductive Health and Research Department (RHR) and the Research Ethics Review Committee of WHO. The protocols are freely available on the WHO website. Building on these achievements, RHR plans to conduct independent multicountry evaluations of existing promising tests and technologies starting from 2017, as an essential next step to advance the understanding of performance, feasibility, acceptability and utility of existing STI POCTs, to develop an investment case for the implementation of STI POCTs within national STI control and prevention programmes and to inform and promote the development of appropriate STI POC diagnostic platforms.

High-quality evaluations in laboratory and real-world settings include evaluating sensitivity and specificity, and predictive values, and determining optimal operational characteristics of STI POCTs in sample sets from high-prevalence and low-prevalence clinical settings and in a variety of populations, for both screening for asymptomatic STIs and for case management. ${ }^{28}$ The clinical utility should be comprehensively evaluated, by morbidity/mortality and adverse event profiles of the target infections.

The IEG emphasised that the required level of sensitivity depends on the context of the site, including among others access, laboratory infrastructure and quality assurance. The evaluations should be carried out opportunistically and take advantage of having a rapid turnaround time for test results to determine whether the POCT can be used to streamline patient pathways and improve the efficiency of the healthcare system. ${ }^{29}{ }^{30}$ The ecology of the site should be comprehensively assessed. The paper on clinic-based evaluation of syphilis POCTs in Verona, included in this supplement, suggested that despite 


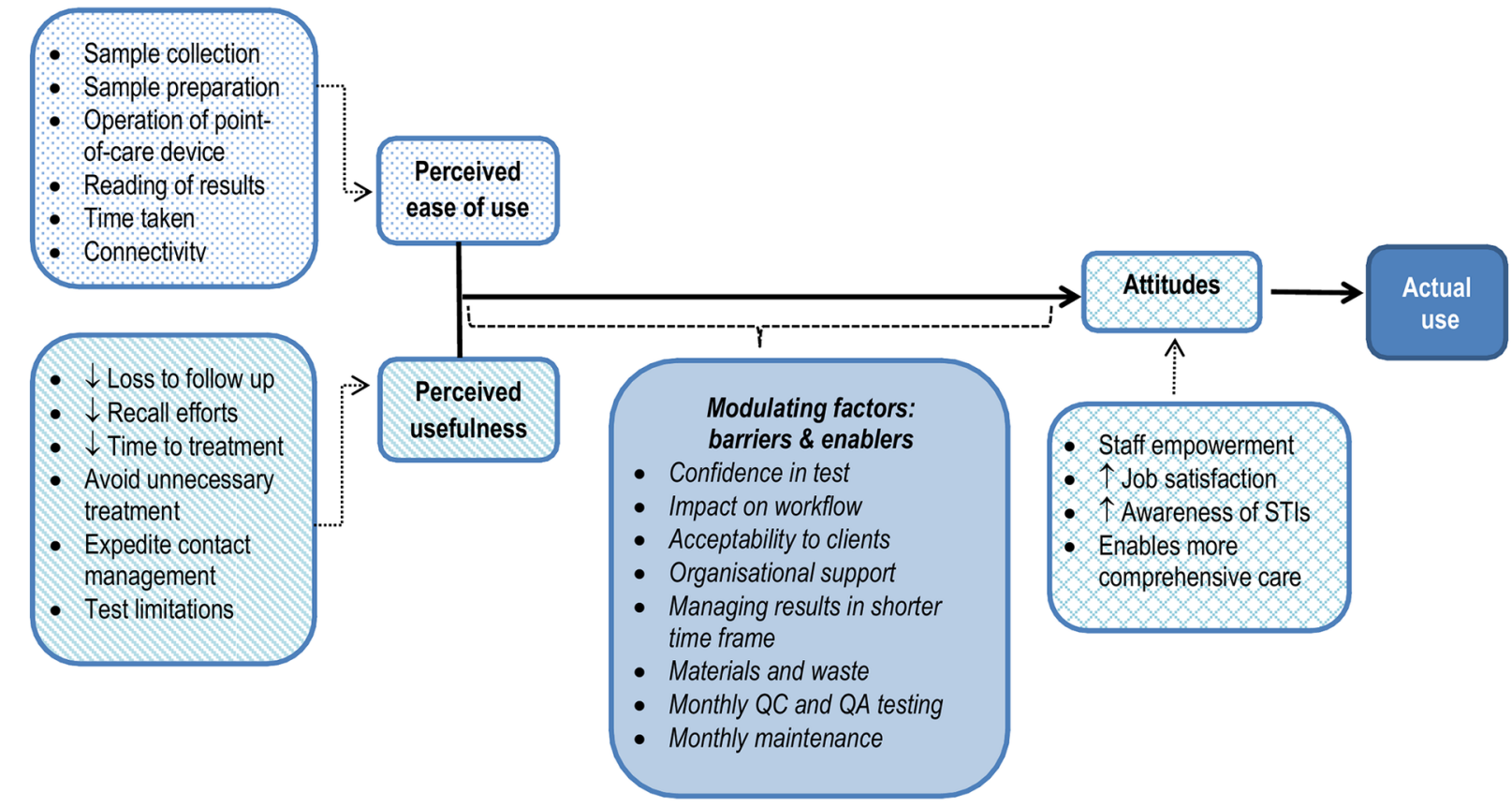

Figure 2 Potential influences on acceptability of a POCT to operators in remote health services. Reprinted from "I Do Feel Like a Scientist at Times": A Qualitative Study of the Acceptability of Molecular Point-Of-Care Testing for Chlamydia and Gonorrhoea to Primary Care Professionals in a Remote High STI Burden, by Natoli et al. ${ }^{31}$ All PLOS content is available under CC BY (Creative Commons Attribution).

low sensitivity, lower than the minimum set in the TPPs, predictive values can still be acceptable for rolling out POCT testing.

Specifically for syphilis POCTs, the experts suggested that the results of evaluations in ANC should be used to reconsider the treatment algorithm. In some settings, the patients might not come back for the result of the reference test and consequent treatment. If the risk of missing to treat a syphilis case is high, then presumptive treatment should be considered. The IEG also raised the issue of including the partners of pregnant women in the evaluations.

The evaluations should also help to clarify what the minimal time should be between the POCT and the (potential) infection and when to retest after risk or treatment.

To accelerate the introduction of promising tests, they should be evaluated before they become commercially available.

Lastly, the IEG noted that conducting POCT evaluations can be a vehicle to assess the quality of participating laboratories and provide capacity building if needed.

Determine local preferences and perceptions of patients and providers towards STI POCTs

The IEG called for more studies to determine local preferences and perceptions on the use of STI POCTs in clinic-based and community-based outreach settings. Qualitative studies should assess attitudes, the perceived usefulness and changes in the management of STIs, which might result in more timely and targeted treatment, earlier commencement of partner notification and reduced follow-up efforts. Providers should be questioned about their confidence in POCT results and consequent treatment decisions and changes in job satisfaction. As shown in figure 2, even though POCTs can shorten episodes of care, managing positive test results in a shorter time frame can also be challenging. ${ }^{31}$

\section{PROGRAMME}

Gaps

The IEG pointed to the lack of evidence for the potential programmatic impact of STI POCTs. Programme science should help to address the gap between the evidence of acceptable test performance and programme design, and assessing how implementation and evaluation of test deployment effectively answers public health needs and improves patient outcomes. $^{32}$

Guidelines, recommendations and regulatory approval for POCTs are scattered, impeding systematic inclusion of POCTs in policies as well as building a strong investment case. The few cost and cost-effectiveness publications about POC testing for STIs are encouraging, but more work should be done on evaluating the actual costs of implementing POCTs tests. ${ }^{33}$

\section{The way forward}

Accelerate the development of STI POCT guidelines and harmonisation of regulatory approval

If POCTs are to be effective STI diagnostics of the future in primary care centres in low- and middle-income countries, there should be a regularly updated set of easy-to-use guidelines and recommendations for use of POCTs for STI case management, screening of asymptomatic infections and surveillance.

WHO has recently published an information note on the use of dual HIV/syphilis rapid diagnostic tests with the main focus on antenatal services. ${ }^{34}$ This is an important step towards the integration of dual HIV/syphilis POCTs into guidelines for 
management of syphilis in pregnant women, aiming to advance the dual elimination of HIV/syphilis.

The IEG suggested a review to determine the regulatory approval frameworks, procurement mechanisms and distributors in settings of interest around the world, this could be informative for both the evaluation of POCTs as for the manufacturers.

\section{Plan for introduction in advance}

The approach to introducing POCTs to healthcare systems should consider all its aspects in advance of product introduction, including patient information, experiences, ideas for uptake, and the clinic and social systems in the local area. Crowdsourcing and using non-traditional ways to include populations in STI POCT provision ${ }^{35}$ might increase community engagement, essential for successful implementation of an STI POCT strategy. ${ }^{36}$

Implementation studies should be conducted to determine staff training needs and inform the development of general and context-specific and population-specific trainings.

\section{Anticipate potential tensions of STI POCT introduction by modelling} the impact on health systems

The technological innovations of POCTs allow for a shift in the health system towards more decentralisation. Decentralised testing has tremendous advantages in accessibility of the services and follow-up, but places considerable pressure on health systems, particularly in terms of personnel, infrastructure and ensuring quality of service. ${ }^{37}$

Moving towards decentralisation of testing could be seen as a concern for future stability by laboratories, as some of their tasks regarding STI-testing would disappear. The EIG suggested assessing the capability and willingness of laboratories to accept a new role: they should monitor and evaluate STI testing both during and after the shift to POCTs. The laboratory should become both a command and training centre for POCTs and their providers. ${ }^{38}$

Harmonisation of critical elements of new pathways to deliver STI POCTs, particularly over-the-counter, should be anticipated. Different public health programmes, for both communicable and non-communicable diseases, should collaborate to harmonise delivery systems for a large spectrum of existing and upcoming POCTs, reducing potential tensions between different parties involved in procurement, delivery, use and postmarket monitoring and evaluation.

If connectivity is adequately implemented, a big part of the disruptive potential of POCT introduction can be minimised. Health systems could improve their efficiency through connectivity solutions, facilitating supply chain management to avoid stock-outs and wastage, and enabling ongoing monitoring of test results, the performance of devices and the competency of the operators from each POCT-site in a country. ${ }^{37}$ The IEG suggested to develop mentorship programmes for both policy makers and programme coordinators.

The IEG acknowledged that the technologies are rapidly changing and opportunities need to be catalysed if manufacturers are to produce the STI POCTs that are needed. Experts urged WHO to bring manufacturers and the wider clinical community together, to guide development, condition funding and negotiate the cost.

Notwithstanding the relative scarcity of available STI POCTs, few companies have commercialised products developed by scientists in academia and industry. Clinicians and industry have different perceptions about the limitations of current tests.
Therefore, industry needs to be more mindful of the requirements of clinicians to introduce marketable tests. ${ }^{13}$

The cost-effectiveness of introducing a new POCT and the potential gains in public health and economic gains should be modelled, considering downstream sequelae. Opportunities for investments and potential returns from investments in STI POCTs should be identified. The development of an investment case should be prioritised as an essential step to engage relevant stakeholders throughout all phases of the diagnostic development cycle. Public health authorities should be engaged to build support for sustainable funding of POCTs as a programmatic goal to strengthen their health system. ${ }^{30}$ The IEG stressed the importance for countries to have a generic, crosscutting STI POCT policy, avoiding ad hoc decisions.

In various countries, home-grown solutions are being developed, and should be encouraged, as small to medium enterprises can address challenges such as importation and national distribution.

\section{Encourage investment in STI POCTs}

The IEG expressed the urgent need to communicate the need for POCTs. The global health sector strategy on STIs, endorsed at the 2016 World Health Assembly, creates a unique opportunity to amplify all elements of the current roadmap through a strong call for innovation in STI control and prevention. ${ }^{1}$ Development of partnerships in the STI POCT field and identifying groups or organisations that can be strong champions could work towards the goal of advancing a clear and compelling message for the need for STI POCT investment.

\section{CONCLUSIONS}

The goal of improving care and treatment of patients worldwide is an overarching public health goal. One way to assist in the modernisation of care is the development and implementation of POCTs. These tests could change and improve provider information, reduce the time to effective case management of those

\section{Key messages}

Key priorities for the advancement of development and global uptake of point-of-care tests for STIs.

- Encourage target discovery.

- Encourage integration and miniaturisation of sample preparation and target detection.

- Promote international and multisectorial research cooperation.

- Prioritise the development of miniaturised multiplexable platforms.

- Include researchers, manufacturers and end-users in the translation stage.

- Capitalise on the advances in connectivity for STI-surveillance, testing uptake and quality control.

- Promote standardised high-quality laboratory, clinic-based and utility evaluations.

- Determine local preferences and perceptions of patients and providers.

- Accelerate the development of guidelines and harmonise regulatory approval.

- Plan for introduction in advance.

- Anticipate potential tensions of introduction by modelling the impact on health systems.

- Encourage investment. 
patients with symptoms and increase screening for asymptomatic infections. This is a long-term goal, but the opportunity to lay the ground work is now available.

Meeting participants at the 2014 and 2015 technical consultations outlined the priorities presented here for accelerating and coordinating the development and introduction of POCTs for STIs. These priorities are an initial starting point for a roadmap, which will be evolving as technology and both public health knowledge and information progress. Although information gaps and hurdles remain, the current state of knowledge provides opportunities to advance the public health goal of the control, treatment and prevention of STIs.

At the present time, there is quite a rich pipeline of diagnostic products, but some are stalled early in the development process. There is a need to accelerate the evaluation of new diagnostics by setting up a competent network of evaluation sites ahead of time, harmonise regulatory approval processes with development of models to estimate cost-effectiveness informed by better STI-data. This should result in accelerated policy development. Although it may be some time before good POCTs can be widely implemented in developing country settings, it is important to advocate for continued development and use of these essential tools as an integral part of both the WHO STI strategy and the agenda for 2030 and have a real impact on the lives of people globally.

Acknowledgements The participants of the meetings were: Moazzam Ali (WHO, Geneva, Switzerland), David Anderson (Burnet Institute, Melbourne, Australia), Manju Bala (VMMC \& Safdarjung Hospital, New Delhi, India), Ron Ballard, Karel Blondeel (WHO, Geneva, Switzerland), Viviane Bremer (ECDC, Berlin, Germany), Nathalie Broutet (WHO, Geneva, Switzerland), Jordi Casabona (CEEISCAT, Barcelona, Spain), Philip Castle (Global Coalition against Cervical Cancer, Arlington, USA), Fatim Cham-Jallow (Makerere University, Kampala, Uganda), Xiang-Sheng Chen (Chinese Academy of Medical Sciences, Nanjing, China), Joanna Cordero (WHO, Geneva, Switzerland), Antonina Crucitti (Institute of Tropical Medicine, Antwerp, Belgium), Carolyn Deal (NIH, Maryland, USA), David Dolinger (FIND, Geneva, Switzerland), Yetunde Fakile (CDC, Atlanta, USA), Tim Farley (Sigma3 Services Sàrl, Nyon, Switzerland), Mario Festin (WHO, Geneva, Switzerland), Miriam Franchini (Brazilian National STI AIDS and Hepatites Program, Brasilia, Brazil), Sebastian Fuller (Public Health England, London, UK), Patricia Garcia (Cayetano Heredia University, Lima, Peru), Charlotte Gaydos (Johns Hopkins University, Baltimore, USA), Sami Gottlieb (WHO, Geneva, Switzerland), Vadum Govorun (Institute of PhysicoChemical Medicine, Moscow, Russian Federation), Catherine Ison (Public Health England, London, UK), Rita Kabra (WHO, Geneva, Switzerland), Mary Kamb (CDC, Atlanta, USA), Kevin Karem (CDC, Atlanta, USA), Maura Laverty (WHO, Geneva, Switzerland), James Kiarie (WHO, Geneva, Switzerland), David Lewis (National Institute for Communicable Diseases, Johannesburg, South Africa), David Mabey (London School of Hygiene and Tropical Medicine, London, UK), Jessica Markby (WHO, Geneva, Switzerland), Natalie Maurer (WHO, Geneva, Switzerland), Oleg Medvedev (Lomonosov Moscow State University, Moscow, Russian Federation), Mario Merialdi (former WHO, Geneva, Switzerland), Robyn Meurant (WHO, Geneva, Switzerland), Maurine Murthagh (International Diagnostics Centre, Woodside, USA), Francis Ndowa (former WHO, Geneva, Switzerland), Lori Newman (WHO, Geneva, Switzerland), Rosanna Peeling (London School of Hygiene \& Tropical Medicine, London, UK), Maria Perez-Gonzales (WHO, Geneva, Switzerland), Tariq Sadiq (University of London, UK), Anita Sands (WHO, Geneva, Switzerland), Adele Schwartz Benzaken (National AIDS Programme, Brazil), Mark Shepard (Flinders University, Adelaide, Australia), Samuel Sia (Columbia University, New York, USA), Marleen Temmerman (WHO, Geneva, Switzerland), Igor Toskin (WHO, Geneva, Switzerland), Thierry Troussier (UNESCO Chair Sexual Health and Human Rights, Paris, France), Ozge Tuncalp Mingard (WHO, Geneva, Switzerland), Magnus Unemo (Örebro University Hospital, Sweden), Sheryl Vanderpoel (WHO, Geneva, Switzerland), Brenda Waning (UNITAID, Geneva, Switzerland), Anna Whalen (FIND, Geneva, Switzerland) and Teodora Wi (WHO, Geneva, Switzerland).

Contributors IT and KB designed the concept and methodology; IT, KB, CD, RWP and JK conducted the analysis and synthesis of the results. All authors contributed to the finalisation of the manuscript.

Disclaimer Disclaimer The authors are staff members of the World Health Organization. The authors alone are responsible for the views expressed in this publication and they do not necessarily represent the views, decisions or policies of the World Health Organization.

Competing interests None declared.
Provenance and peer review Commissioned; externally peer reviewed.

Open Access This is an Open Access article distributed in accordance with the Creative Commons Attribution Non Commercial (CC BY-NC 4.0) license, which permits others to distribute, remix, adapt, build upon this work non-commercially, and license their derivative works on different terms, provided the original work is properly cited and the use is non-commercial. See: http://creativecommons.org/ licenses/by-nc/4.0/

(c) World Health Organization 2017. Licensee BMJPublishing Group Limited. This is an open access articledistributed under the terms of the Creative CommonsAttribution IGO License (https:// creativecommons.org/licenses/by/3. 0/ igo), which permits use, distribution, and reproduction for non-commercial purposes in anymedium, provided the original work is properly cited. Inany reproduction of this article there should not be anysuggestion that WHO or this article endorse any specificorganization or products. The use of the WHO logo isnot permitted. This notice should be preserved alongwith the article's original URL.

\section{REFERENCES}

1 World Health Organization. Global health sector strategy on sexually transmitted infections, 2016-2021. Geneva: WHO, 2016. http://www.who.int/reproductivehealth publications/rtis/ghss-stis/en/

2 Hsieh YH, Gaydos CA, Hogan MT, et al. Perceptions on point-of-care tests for sexually transmitted infections - comparison between frontline clinicians and professionals in industry. Point Care 2012;11:126-9.

3 Gottlieb SL, Deal CD, Giersing B, et al. The global roadmap for advancing development of vaccines against sexually transmitted infections: update and next steps. Vaccine 2016:34:2939-47.

4 Broutet N, Fruth U, Deal C, et al. On behalf of participants of the 2013 STI vaccine technical consultation. Vaccines against sexually transmitted infections: the way forward. Vaccine 2014;32:1630-7.

5 Peeling RW, Holmes KK, Mabey D, et al. Rapid tests for sexually transmitted infections (STIs): the way forward. Sex Transm Infect 2006;82:v1-v6.

6 Pai NP, Vadnais C, Denkinger C, et al. Point-of-care testing for infectious diseases: diversity, complexity, and barriers in low- and middle-income countries. PLOS Med 2012;9:e1001306.

7 Shephard M. Point-of-care testing comes of age in Australia. Aust Prescr 2010;33:6-91.

8 World Health Organization. Global Strategy for the prevention and control of sexually transmitted infection. Geneva: WHO, 2007

9 Toskin I, Murtagh M, Peeling RW, et al. Advancing prevention of sexually transmitted infections through point-of-care testing: target product profiles and landscape analysis. Sex Transm Infect 2017;0:1-12.

10 Bristow CC, Dong H, Klausner JD. Technological solutions to address drug-resistant Neisseria gonorrhoeae. Emerg Infect Dis 2016;22:939-40.

11 Buono SA, Watson TD, Borenstein LA, et al. Stemming the tide of drug-resistant Neisseria gonorrhoeae: the need for an individualized approach to treatment. J Antimicrob Chemother 2015:70:374-81.

12 Park S, Zhang Y, Lin S, et al. Advances in microfluidic PCR for point-of-care infectious disease diagnostics. Biotechnol Adv 2011;29:830-9.

13 Spindel S, Sapsford KE. Evaluation of optical detection platforms for multiplexed detection of proteins and the need for point-of-care biosensors for clinical use. Sensors 2014;14:22313-41.

14 Tariq S, Mazzaferri F, Unemo M. Rapid accurate point-of-care tests combiningdiagnostics and antimicrobial resistance prediction for Neisseria gonorrhoeae and Mycoplasmagenitalium. Sex Transm Infec 2017;0:1-4

15 Luppa PB, Bietenbeck A, Beaudoin C, et al. Clinically relevant analytical techniques, organizational concepts for application and future perspectives of point-of-care testing. Biotechnol Adv 2016:34:139-60.

16 Wedderburn CJ, Murtagh M, Toskin I, et al. Using electronic readers to monitor progress toward elimination of mother-to-child transmission of HIV and syphilis: an opinion piece. Int J Gynaecol Obstet 2015;130(Suppl 1):S81-S83.

17 Chin CD, Laksanasopin T, Cheung YK, et al. Microfluidics-based diagnostics of infectious diseases in the developing world. Nat Med 2011;17:1015-9.

18 Vashist SK, Luppa PB, Yeo LY, et al. Emerging Technologies for Next-Generation Pointof-Care Testing. Trends Biotechnol 2015;33:692-705.

19 Garcia PJ, You P, Fridley G, et al. Point-of-care diagnostic tests for low-resource settings. Lancet Glob Health 2015;3:e257-e258.

20 Hsieh YH, Gaydos CA, Hogan MT, et al. What qualities are most important to making a point of care test desirable for clinicians and others offering sexually transmitted infection testing? PLoS One 2011;6:e19263.

21 Hsieh YH, Hogan MT, Barnes M, et al. Perceptions of an ideal point-of-care test for sexually transmitted infections--a qualitative study of focus group discussions with medical providers. PLoS One 2010;5:e14144.

22 Rompalo AM, Hsieh YH, Hogan T, et al. Point-of-care tests for sexually transmissible infections: what do 'end users' want? Sex Health 2013:10:541-5.

23 Cheng B, Cunningham B, Boeras DI, et al. Data connectivity: a critical tool for external quality assessment. Afr J Lab Med 2016;5:a535. 


\section{Original article}

24 Kersaudy-Rahib D, de Barbeyrac B, de Diego S, et al. Home screening compared with clinic-based screening for Chlamydiae trachomatis in France: a randomised controlled trial. The Lancet 2013;382(S3):S53.

25 Gibbs J, Gkatzidou V, Tickle L, et al. 'Can you recommend any good STI apps?' A review of content, accuracy and comprehensiveness of current mobile medical applications for STIs and related genital infections. Sex Transm Infect 2017;93:234.1-5.

26 Elliot $\mathrm{E}$, Rossi M, McCormack S, et al. Identifying undiagnosed HIV in men who have sex with men (MSM) by offering HIV home sampling via online gay social media: a service evaluation. Sex Transm Infect 2016;92:470-3.

27 Banoo S, Bell D, Bossuyt P, et al. Evaluation of diagnostic tests for infectious diseases: general principles. Nat Rev Microbiol 2010;8(12 Suppl):S17-29.

28 Dallabetta G, Tec W, Nielsen G, et al. Prevention and control of STD and HIV infection in developing countries. In: Holmes KK, Sparling P, Stamm W. Sexually transmitted diseases. 4, 2008.

29 Mabey DC, Sollis KA, Kelly HA, et al. Point-of-care tests to strengthen health systems and save newborn lives: the case of syphilis. PLoS Med 2012;9:e1001233.

30 García PJ, Cárcamo CP, Chiappe M, et al. Rapid syphilis tests as catalysts for health systems strengthening: a case study from Peru. PLoS One 2013:8:e66905.

31 Natoli L, Guy RJ, Shephard M, et al. 'I do feel like a scientist at times': a qualitative study of the acceptability of molecular point-of-care testing for Chlamydia and
Gonorrhoea to primary care professionals in a remote high STI burden setting. PLOS One 2015;10:e0145993.

32 Peeling RW, Mabey D, Ballard RC. Introducing new diagnostics into STI control programmes: the importance of programme science. Sex Transm Infect 2013:89:115-9.

33 Herbst de Cortina S, Bristow CC, Joseph Davey D, et al. A systematic review of point of care testing for Chlamydia trachomatis, Neisseria gonorrhoeae, and Trichomonas vaginalis. Infect Dis Obstet Gynecol 2016;2016:1-17.

34 World Health Organization. Information note on the use of dual HIV/syphilis rapid diagnostic tests (RDT). Geneva: WHO, 2017.

35 World Health Organization. Guidelines on hepatitis B and C testing. Geneva: WHO, 2017. http://www.who.int/hepatitis/publications/guidelines-hepatitis-c-b-testing/ en/

36 Aicken CR, Fuller SS, Sutcliffe LJ, et al. Young people's perceptions of smartphoneenabled self-testing and online care for sexually transmitted infections: qualitative interview study. BMC Public Health 2016;16:974.

37 Nkengasong J, Boeras DI, Abimiku Alash'le, et al. Assuring the quality of diagnostic testing: the future is now. Afr J Lab Med 2016;5:a558.

38 Boeras DI, Nkengasong JN, Peeling RW. Implementation science: the laboratory as a command centre. Curr Opin HIV AIDS 2017;12:171-4. 